\section{Staatliche Förderung von Großtechnikprojekten. Ein dys- funktionaler Anachronismus im Zeitalter der Globalisierung?}

\author{
von Johannes Weyer, Universität Dortmund
}

\begin{abstract}
Obwohl staatlich geförderte Großtechnikprojekte häufig gescheitert sind, erfreut sich dieser Politiktyp nach wie vor einer großen Beliebtheit, sowohl im nationalen als auch im internationalen Rahmen. Der Beitrag argumentiert, dass man die politische Logik der Entscheidungsprozesse beleuchten muss, um das - scheinbar irrationale - Handeln der Akteure zu verstehen. Dabei bleibt der Nationalstaat der Referenzrahmen, in dem die Gewinne ausgezahlt werden, die sich in Machtspielen in transnationalen Verhandlungsprozessen ergeben.
\end{abstract}

\section{Einleitung}

Die Frage nach dem Stellenwert der (national-) staatlichen Innovationspolitik in der globalen Wissensgesellschaft beantwortete Bundesverkehrsminister Manfred Stolpe am Tag der Einführung der satellitengestützten Lkw-Maut in Deutschland mit einer Formulierung, die aus den 1980er Jahren hätte stammen können: Deutschland habe sich mit der Entwicklung des Maut-Systems ,einen Wettbewerbsvorteil erarbeitet“ und damit zur „Stärkung des Standorts Deutschland durch technologische Innovation“ beigetragen, was zudem „neue Exportchancen“ eröffne (BMVBW 2005). Und nahezu wortgleich findet man in Verlautbarungen von Forschungsministerin Edelgard Bulmahn zur deutschen Raumfahrtpolitik bekannte Formulierungen von der ,weltweit herausragenden Position", die Deutschland in der Raumfahrt einnimmt; die dabei erbrachten „technologischen Leistungen“ seien „ein wichtiger Faktor für unser hohes Ansehen als HochtechnologieStandort“, so die Ministerin weiter (BMBF 2004, S. 5). Die offen propagierte Einbettung der Innovations- und Technologiepolitik in Strategien nationalstaatlicher Profilierung wird seit einigen Jahren durch den Hinweis verstärkt, dass der „uneingeschränkte Zugriff“ auf Satellitensysteme für die Erdbeobachtung „zugleich unsere außenpolitische Handlungsfä- higkeit“ sichere (ebd.). Milliardenschwere Investitionen in Großprojekte der Raumfahrt, die Bulmahn noch vor wenigen Jahren vehement kritisiert hatte, erhalten durch diese Indienstnahme für die neue, selbstbewusste Außenund Sicherheitspolitik der Berliner Republik eine überraschende neue Legitimation.

\section{Großtechnik - ein Auslaufmodell?}

Die staatliche Förderung von Großtechnikprojekten, die in den 1940er Jahren mit dem Bau der V-2-Rakete im Nazi-Deutschland sowie der Atombombe in den USA begann, ist also keineswegs ein Auslaufmodell, wie es beispielsweise Ulrich Dolata mit seiner These vom ,sukzessiven Rückzug des Staates aus der großtechnologischen Forschungsförderung“" (Dolata 2004, S. 26) und dessen Hinwendung zum neuen Typus der „unfassbaren Technik“ (S. 9) nahe legt. Im Gegenteil: Die Liaison von Staat und Großtechnik scheint - zumindest in Deutschland - sogar enger zu werden, da seit Mitte der 1990er Jahre die Außen- und Sicherheitspolitik neben der Wissenschaftsförderung (1960er/70er Jahre) und der Industriepolitik (1980er/90er Jahre) als neues Begründungsmuster für das staatliche Engagement in der Entwicklung großtechnischer Systeme ins Feld geführt wird. Auch wenn eine Verlagerung von Kompetenzen und Projektträgerschaften auf multinationale Organisationen kaum zu bestreiten ist, wäre es angesichts der ungebrochenen Tradition einer Indienstnahme von Technik für politische Zwecke verfehlt, einen Bedeutungsverlust des Nationalstaates in der Innovationspolitik zu diagnostizieren, wie es beispielsweise Edgar Grande tut (2001). Denn der Nationalstaat bildet nach wie vor das Referenzsystem, innerhalb dessen die Gewinne ausgezahlt werden, die aus den Machtspielen resultieren, die nicht nur im nationalen Rahmen, sondern in zunehmendem Maße auch in multinationalen Strukturen bzw. in Mehrebenen-Systemen stattfinden.

Zudem bestehen nach wie vor hohe Erwartungen an den Staat als die Instanz, die angesichts des sich verschärfenden internationalen Standortwettbewerbs - für die Zukunftssicherung sowie die Schaffung geeigneter Rahmenbedingungen für die Ausschöpfung von Innovationspotenzialen zuständig ist. Dass staatliche Akteure diese Aufgaben in komple- 
xen, multinationalen Konstellationen vollbringen, ändert nichts an der Tatsache, dass ihre Leistungen immer noch anhand von Kennziffern vermessen werden, die die ,technologische Leistungsfähigkeit einer Volkswirtschaft" (BMBF 2004, S. 474) ausdrücken und als Berechnungsgrundlage den Nationalstaat und als Vergleichsmaßstab die konkurrierenden, hoch entwickelten Industriestaaten verwenden.

\section{Raumfahrt als Prototyp}

In gewisser Weise kann die Raumfahrt als ein früher Vorreiter des neuartigen Politik-Typus angesehen werden, der durch eine MehrebenenStruktur sowie durch eine virulente und konflikthafte Mischung von Kooperation und Konkurrenz gekennzeichnet ist (und zugleich eine lange Tradition der Orientierung auf Großtechnikprojekte aufweist). Die europäische Raumfahrtorganisation (ESRO/ELDO, später ESA), die zweite große multinationale Forschungsorganisation Europas nach dem Kernforschungszentrum CERN, war seit ihrer Gründung in den frühen 1960er Jahren stets durch die divergierenden Interessen der beteiligten Nationen (insbesondere Frankreichs, Deutschlands und Großbritanniens) geprägt, die oftmals nur durch mühsam erzielte und fragile Kompromisse überbrückt werden konnten. Allen Partnern war stets bewusst, dass das Ziel einer eigenständigen, d. h. von den USA unabhängigen europäischen Raumfahrt nur gemeinsam erreicht werden konnte; doch allen Beteiligten war zugleich klar, dass jeder Partner eigene Ziele verfolgte und die Kooperation oftmals instrumentell handhabte, d. h. darauf bedacht war, so viel Gewinn wie möglich aus den Gemeinschaftsprojekten zu ziehen. Die Beteiligung Deutschlands am Bau der Europa-Rakete in den 1960er Jahren war beispielsweise ein geschickter Schachzug der Franzosen, das wieder erwachende TechnologiePotenzial der Deutschen unter Kontrolle zu bekommen und zugleich eine TechnologieEntwicklung zu europäisieren, d. h. von den Partnern mit finanzieren zu lassen, die für die Aufrechterhaltung des Weltmacht-Anspruchs Frankreichs unentbehrlich war (dazu ausführlich Weyer 1993a, S. 215-325).

Eine echte Verlagerung von Kompetenzen auf die multinationale Organisation (im Sinne einer Preisgabe von industriellen Entwick- lungskapazitäten oder politischen Steuerungsinstrumenten) hat jedoch nicht stattgefunden. Im Gegenteil: Die jahrzehntelang andauernden Kontroversen um die Prinzipien des „Fair return“ verdeutlichen, dass die prekäre Balance zwischen Gemeinschafts-Vorhaben und nationalen Rückflüssen stets ein Feld für Interessenkonflikte und Machtspiele war. Eine der Hauptleistungen der ESA ist, dass sie bereits in den 1970er Jahren Verfahren und Instrumente zur Ausbalancierung derartiger Konflikte gefunden hat, die in anderen Bereichen der europäischen Politik erst noch geschaffen werden müssen (vgl. Reinke 2004, S. 134 f.).

Allerdings finden auch in der ESA in steter Regelmäßigkeit Macht-Spiele mit bekannten strukturellen Konstellationen statt: In den letzten Jahren ging es insbesondere um eine stärkere Verzahnung von ESA und EU, um im Zeitalter globaler Krisenintervention eine Neuausrichtung der europäischen Raumfahrtkapazitäten auf außen- und sicherheitspolitische Anwendungen zu ermöglichen (vgl. Hagen, Scheffran 2000). Ein zweiter Schwerpunkt ist erneut ein Großtechnikprojekt, und zwar das europäische Satellitennavigations-System Galileo, das zum Preis von 3,4 Mrd. Euro bis zum Jahr 2008 im All stationiert werden und eine Alternative zum amerikanischen GPS-System bieten soll - mit einer Vielzahl von Nutzanwendungen beispielsweise im Bereich der Verkehrstelematik. Der Entscheidungsprozess war von langwierigen Auseinandersetzungen zwischen Italien und Deutschland um die „Systemführung“ begleitet (während andere Länder das Projekt insgesamt ablehnten). Auch in diesem Kooperationsprojekt spielten nationale Aspekte wie der „Führungsanspruch" (FAZ 28.05.2003, S. 11) eines Landes eine zentrale Rolle; und der Startschuss für das Projekt konnte im Jahr 2002 erst gegeben werden, nachdem Deutschland sich durchgesetzt hatte. Forschungsministerin Bulmahn drückte die politische Logik derartiger Aushandlungsprozesse in bemerkenswerter Offenheit aus:

„... die Raumfahrt hat für uns nicht nur eine wissenschaftliche und eine wirtschaftliche Dimension, sondern vor allem auch eine politische.“ (Bulmahn 2002, S. 5)

Angesichts des verhaltenen Interesses der Industrie am Galileo-Projekt stellt sich die Frage, wieso Politiker jeglicher parteipolitischer Couleur immer wieder technologisch visionäre 
Großprojekte initiieren und unter Einsatz von Steuergeldern vorantreiben, die mit großen Unsicherheiten behaftet sind und deren wirtschaftlicher Nutzen zweifelhaft ist, die jedoch eine offenkundige politische Dimension besitzen. (So liegt der militärische Nutzen des GalileoSystems für eine europäische Eingreiftruppe auf der Hand - und dies wird auf EU-Ebene auch offen diskutiert.) Galileo ist das jüngste Produkt in der langen Liste der (meist gescheiterten) Projekte, die auf einer Innovations-Strategie basieren, welche dem Staat industriepolitische Funktionen sowie die Rolle des Technologietreibers in Hochtechnologiefeldern zuschreibt.

Es wäre also zu klären, warum die Akteure des politischen Systems eine derartige Affinität zu Großtechnik-Projekten haben (Kap. 4) und welche Strategien sie mit deren Förderung verbinden (Kap. 7).

\section{Die symbiotische Entwicklung von (Groß-) Technik und modernem Interventionsstaat}

In Anlehnung an Renate Mayntz sollen unter den Begriff „große technische Systeme“ die drei Typen „Großanlagen“ (z. B. Kernkraftwerke), „Großvorhaben“ (z. B. Manhattan Project) sowie ,große technische Infrastruktursysteme" etwa in den Bereichen Telekommunikation oder Verkehr subsummiert werden (Mayntz 2001, S. 5). Prinzipiell gibt es zwei Wege, diesen etwas unscharfen Begriff näher zu bestimmen. Den ersten beschreiten Nils Bruzelius et al., die „Mega-Projekte“ anhand folgender quantitativer Parameter charakterisieren: Höhe der Investitionen, lange Lebensdauer, hohes Maß an Unsicherheit sowie indirekter Nutzen, der vom Betreiber nicht internalisiert werden kann (Bruzelius et. al 2002, S. 144). Der offenkundige Nachteil dieser Begriffsbestimmung ist, dass es keine eindeutigen und unstrittigen Schwellenwerte für die „Größe“ eines technischen Systems gibt.

Den zweiten Weg beschreitet Mayntz, die die Entwicklung großer technischer Infrastruktursysteme modernisierungstheoretisch deutet und dabei postuliert, dass deren Verbreitung und Nutzung die Ausdifferenzierung der Teilsysteme moderner Gesellschaften maßgeblich „beeinflusst“ (Mayntz 2001, S. 5) haben. Sie verweist auf die „komplexe Interdependenz“ von Technikentwicklung und Herausbildung des modernen Staates, bei der insbesondere „die Militärtechnik und die modernen technischen Infrastruktursysteme eine entscheidende Rolle“ (S. 15) spielten. Dabei habe sich eine gewisse strukturelle Isomorphie derart ergeben, dass „der moderne Zentralstaat und die großen technischen Systeme“ sich nicht nur „wechselseitig in ihrem Wachstum stimuliert", sondern darüber hinaus auch ,in ihrer Zentralisierung gefördert“ (ebd.) haben. Der staatlich geförderte Auf- und Ausbau industrieller Monopole sowie der Betrieb von Infrastruktursystemen in staatlicher Regie sind Indizien für diese engen Beziehungen von Großtechnik und modernem Interventionsstaat (S. 16). Allerdings verweist Mayntz auch darauf, dass „die auf einer Art struktureller Wahlverwandtschaft gestützte Symbiose zwischen hierarchisierten politischen, industriellen und technischen Systemen“ angesichts der Tendenzen zur Dezentralisierung sowie des erodierten Vertrauens in Technik mittlerweile „prekär“ (ebd.) geworden ist.

Es gibt also starke und durch eine Vielzahl empirischer Studien fundierte Indizien für die These, dass die Ausbildung des modernen Interventionsstaates in einem engen Zusammenhang mit der Entwicklung großer technischer Systeme (sowie der sie produzierenden Industrie) gesehen werden muss. Dass derartige Konstellationen soziale Eigendynamiken produzieren, die außer Kontrolle geraten und zu Sachzwängen gerinnen können, hat Dwight D. Eisenhower 1958 erstmals in der - von Mayntz wieder aufgegriffenen - Formel vom „militärisch-industriellen Komplex“ ausgedrückt (vgl. Weyer 1999).

Die besondere Affinität staatlicher Akteure zur Großtechnik wäre vor diesem historischen Hintergrund also erklärbar als ein Rückgriff auf die gesellschaftlichen Ressourcen, die bis in die jüngste Vergangenheit stets ein probates Instrument zur Etablierung, Aufrechterhaltung und Ausdehnung des Macht- und Zuständigkeitsbereichs des politischen Systems waren. Ob dieser Mechanismus angesichts der aktuellen - durchaus widersprüchlichen - Tendenzen von Dezentralisierung und Zentralisierung durch neue Muster abgelöst wird, ist m. E. eine offene Frage. Es spricht allerdings viel dafür, dass einerseits die von Kompetenzverlust bedrohten nationalstaatlichen Akteure das bewährte Instrumentarium z. B. der Förderung großtechnischer Projekte 
nutzen werden, um ihre Macht zu erhalten, und zugleich die von Kompetenzzuwächsen profitierenden Akteure (z. B. auf EU-Ebene) ähnliche Instrumente einsetzen werden wie zuvor der Nationalstaat. Dies wäre beispielsweise eine plausible Erklärung für das Galileo-Projekt und die politische Logik der damit verbundenen Entscheidungsprozesse.

\section{Charakteristika von Großtechnik}

Kombiniert man die Ansätze von Bruzelius et al. und Mayntz, so gelangt man zu einer Definition von Großtechnik, welche die Variablen „Größe/Unsicherheit“ und „Handlungsfähigkeit/Kompetenzausbau des politischen Systems“ integriert. Unter den Begriff „Großtechnik“ fallen demzufolge Projekte, Anlagen oder Netzwerke einer gewissen, jedoch nicht präzise quantifizierbaren Größenordnung, die auf die Erzeugung innovativer, aber marktferner Techniken ausgerichtet und mit einem hohem Maß von Unsicherheit behaftet sind (Beispiele: Apollo-Projekt, Schneller Brüter, Transrapid, Internationale Raumstation). Die Nutzungsvisionen sind in der Regel primär von politischen Zielen und Motiven geprägt (militärische Stärke, Energieversorgung, Verkehrspolitik etc.), die bei den Marktakteuren - abgesehen von den unmittelbaren Nutznießern der Projekte - kaum Resonanz erzeugen, sondern meist Desinteresse und verhaltene Skepsis, z. T. sogar offene Ablehnung hervorrufen. Die Finanzierung der Projekte sowohl in der Entwicklungs- als auch in der Betriebsphase liegt meist vollständig in der Hand des Staates, der dabei auf eine - von ihm genährte - Industrielobby angewiesen ist, zu der sich ein klientelistisches, symbiotisches Verhältnis der gegenseitigen Abhängigkeit entwickelt. Der politisch-symbolische Nutzen, insbesondere der Macht- und Prestigegewinn des Staates dominiert meist gegenüber dem wirtschaftlichen Nutzen derartiger Großtechnikprojekte.

Diese Interpretation von Großtechnik als eines machtpolitischen Instruments moderner Staaten hat jedoch eine offenkundige „Schwachstelle“, denn die erwarteten Effekte im politischen System werden mit gewissen Risiken erkauft, die mit gewagten und visionären Großtechnikprojekten typischerweise einhergehen. Diese Risiken entstehen zum einen aus der mangelnden Kalkulierbarkeit des Erfolgs sowie der möglichen Nebenwirkungen großtechnischer Projekte (z. B. die Proliferation militärisch sensitiver Raketentechnik), zum anderen aus den manifesten Risiken von Fehlschlägen von Hochrisikotechnologien (z. B. der GAU eines Kernkraftwerks). Es erscheint somit erklärungsbedürftig, warum Politiker in ihrem Bestreben nach Machtzuwachs ausgerechnet auf einen Typus von Technik setzen, der offenbar schwer kontrollierbar ist, unerwünschte und unkontrollierbare Effekte generiert und immer wieder spektakuläre (und gesellschaftlich folgenreiche) „Flops“ und Fehlschläge produziert.

\section{Policy Failure}

Die Liste der gescheiterten Großtechnikprojekte ist lang, und sie werden in der Fachliteratur meist als ein Indiz für Staatsversagen (,policy failure“) interpretiert (vgl. u. a. Keck 1984, Bruzelius 2002). Bereits die V-2-Rakete war ein sinnloses und irrationales Projekt, das jeglicher militärischer Logik widersprach, weil die enormen Kosten in keinem Verhältnis zum geringen militärischen Nutzen standen. Eine unabhängige Bewertung der Leistungsfähigkeit der V-2 wurde nie vorgenommen; und die Fortführung des Projekts bis in das Jahr 1945 verdankt sich neben dem organisationalen Eigeninteresse der Heeresversuchsanstalt Peenemünde vor allem der institutionellen Konstellation der Nazi-Politik sowie der von Hitler, Himmler und anderen gehegten - und von Wernher von Braun taktisch geschickt genährten - Hoffnung auf die ultimative Waffe (als technisches Mittel zur Lösung militärisch-politischer Probleme, vgl. Weyer 1999).

Der Schnelle Brüter (Keck 1984), der Raumtransporter Sänger (Weyer 1992) und die Magnetbahn Transrapid (Weyer 1997) sind weitere, staatlich initiierte und geförderte Großprojekte, die entweder ganz aufgegeben werden mussten oder aber die in sie gesetzten Hoffnungen nicht erfüllten. Aktuelle Beispiele, in denen sich die rot-grüne Bundesregierung ähnlich wie ihre Vorgängerinnen engagiert, sind die Internationale Raumstation ISS, das Lkw-Maut-System von Toll-Collect sowie - damit zusammenhängend - das Navigationssatelliten-System Galileo. Alle drei Systeme wären allein aufgrund unternehmerischer Initiative nie zustande ge- 
kommen; der Staat spielte vielmehr die Rolle des Technologietreibers, der mit massivem Einsatz von Mitteln die Entwicklung visionärer Hochtechnologien vorantrieb, die - im Licht von Alternativen - ein denkbar schlechtes Kosten-Nutzen-Verhältnis aufwiesen. ${ }^{1}$

\section{Die (Dys-)Funktionalität der Großtechnik}

Es gibt zwei unterschiedliche Interpretationsansätze, mit deren Hilfe sich der empirische Befund analysieren lässt, dass der Staat immer wieder gegen alle Vernunft auf Großtechnikprojekte setzt.

\subsection{Die Irrationalität staatlichen Handelns}

Die erste Position konstatiert eine Irrationalität der staatlichen Technologiepolitik und verbindet dies mit einer aufklärerisch-normativen Forderung nach einer rationaleren Politikgestaltung, z. B. durch verbesserte Prognoseinstrumente oder aber durch eine verstärkte Beteiligung von Nutzern und Betroffenen (vgl. z. B. Bruzelius 2002). Diese Position vertritt - explizit oder implizit - ein Modell von Politik, das eine Gemeinwohlorientierung staatlichen Handelns unterstellt und die Qualität politischer Entscheidungen daran misst, in welchem Maße es gelingt, gesellschaftlich konsensfähige Ziele umzusetzen. Staatliche Großprojekte, die gegen alle wirtschaftliche Vernunft sowie den wissenschaftlichen Sachverstand durchgesetzt werden, sind aus dieser Perspektive dysfunktional.

In etlichen Fallstudien ist immer wieder akribisch nachgewiesen worden, dass technologische Großprojekte - entgegen aller Rhetorik oftmals nicht wissenschaftlichen oder ökonomischen Zielen dienen. Zudem profitieren meist einige wenige, mit der Politik symbiotisch verbundene Interessengruppen von der Durchführung der Vorhaben, und die Programmformulierung wird oftmals partiell oder gänzlich an diese Lobby delegiert. Otto Keck (1988) macht zudem auf folgende Informationsasymmetrie aufmerksam: Die staatlichen Akteure haben in der Regel nur unvollständiges Wissen über die Erfolgsaussichten eines Großprojektes, weil sie von der Lobby systematisch im Unklaren gelassen und mit überoptimistischen Prognosen versorgt werden. Einen ähnlichen Mechanismus beschreiben auch Bruzelius et al. (2002), die auf die mangelhafte Überprüfung von Projektvorschlägen verweisen und zudem einen Interessenkonflikt auf
Seiten des Staates diagnostizieren, der bei Großprojekten stets mehrere Rollen zugleich spielt: Er ist Initiator und Finanzier des Projekts, oftmals auch Betreiber der Anlage, übernimmt aber zugleich die Aufgabe der Aufsicht und Regulation. Zudem soll der Staat als Sachwalter öffentlicher Interessen fungieren, was aber in der Gemengelage von Interessen und Rollen oftmals untergeht.

Herbert Simon und andere haben für ein derartiges Entscheidungsverhalten den Begriff der „bounded rationality“ geprägt; und ein Bericht über das Vorgehen des Bundesverkehrsministeriums bei den Verhandlungen mit den Betreibern des Lkw-Maut-Systems liest sich wie eine Fallstudie dieses Theorems: Das BMVBW, so rügt der Bundesrechungshof, habe im „blinden Vertrauen“ auf die Leistungsfähigkeit des Betreiberkonsortiums „auf eine Erprobung des Systems verzichtet“; man habe sich das System nur einmal demonstrieren lassen, danach habe es dann „keine Kontrollen des Fortschritts bei der Entwicklung des Systems ... mehr gegeben“ (zit. n. FAZ.net 26.10.2004).

Aus der Perspektive des Policy-failureModells war dies eine unverantwortliche Schlamperei; doch richtet man den Blick auf die Funktionalität begrenzt-rationaler Entscheidungsprozesse für die Handlungsfähigkeit von Organisationen, so könnte die Überprüfung des Industrie-Partners seitens der Politik auch deswegen unterblieben sein, weil man ansonsten eine wertvolle Ressource verloren hätte, in die man nicht nur eine Menge Geld investiert, sondern von der man auch die eigene Glaubwürdigkeit abhängig gemacht hatte.

\subsection{Die politische Rationalität staatlichen Handelns}

Die zweite Position verfolgt daher einen eher akteur- bzw. organisationstheoretischen Ansatz, der nicht (vermeintlich) objektive Bewertungsmaßstäbe zugrunde legt, sondern die soziale Logik des Handelns der Akteure rekonstruiert und ihre Entscheidungen vor dem Hintergrund eines Choice-Constraint-Modells interpretiert. Hier geht es also eher um die politische Rationalität politischen Handelns, d. h. den (Eigen-) Nutzen für die Akteure des politischen Systems, und weniger um dessen Beitrag zur Steigerung des Gemeinwohls. Die zu Grunde liegende These besagt, dass die Akteure des politischen Sys- 
tems vor allem an den innersystemischen Resonanzen und weniger an den system-externen Effekten ihres Handelns interessiert sind (Weyer 1993b). In diesem Sinne kommt es also weniger auf das (technische) Gelingen von Großprojekten an, sondern auf die Rückwirkungen, die derartige Projekte im politischen System erzeugen. Diese Effekte bestehen vor allem im Domänenerhalt und -ausbau und haben damit einen wesentlich kurzfristigeren Zeithorizont als die oftmals auf Jahrzehnte angelegten Großprojekte. Die Entstehung des Bundesforschungsministeriums BMWF im Jahre 1962 ist ein instruktives Beispiel für diesen Mechanismus der Besetzung einer Domäne mit Hilfe der Großprojekte der europäischen Raumfahrt (vgl. Weyer 1993a).

Nach diesem Verständnis unterliegt also auch - bzw. gerade - die Technologiepolitik einer politischen Logik (vgl. Reinke 2004, S. 370); denn sie ist ein Teilbereich des politischen Systems, in dem die beteiligten Akteure jenseits aller Rhetorik nicht uneigennützig (bzw. gemeinwohlorientiert) agieren, sondern nach der Maxime handeln, ihre Macht zu festigen und ihren Einfluss zu mehren. Das schließt die Gemeinwohlorientierung von Mitgliedern des politischen Systems nicht per se aus, besagt jedoch, dass allein mit Gemeinwohlorientierung keine Politik zu machen ist, d. h. das eigene „Überleben“ im System nicht gewährleistet werden kann. $^{2}$ Großtechnikprojekte eignen sich aufgrund ihrer publizitätswirksamen Sichtbarkeit, ihrer symbolischen Bedeutung sowie ihres Ressourcenbedarfs in besonderem Maße zur Profilierung gegenüber dem politischen Gegenspieler, sei es dem konkurrierenden Ministerium im eigenen Land, dem konkurrierenden nationalstaatlichen Akteur in multinationalen Kooperations-Projekten oder der nächsten Ebene in einem Mehrebenen-Verhandlungssystem. In diesem Sinne ist die Fixierung politischer Akteure auf Großtechnikprojekte durchaus funktional, weil sie die Durchsetzung eigener Strategien befördern kann.

\subsection{Macht-Spiele mit Großtechnik}

Aus dieser akteurtheoretischen Perspektive lassen sich nunmehr zwei bislang ungelöste Fragen beantworten, und zwar die nach der Rolle des Nationalstaats und die nach der spezifischen Riskanz symbiotischer Arrangements von (Macht-)Politik und (Groß-)Technik.
Die Frage nach der Bedeutung des Nationalstaats kann nicht apodiktisch beantwortet werden, denn es hängt immer vom Typus des Machtspiels, d. h. von der spezifischen Akteurkonstellation ab, in der die Gewinne ausgezahlt werden, die sich aus Machtspielen ergeben. Empirisch gesehen, verorten sich die meisten Politik-Akteure nach wie vor in einem nationalstaatlichen Referenzsystem, d. h. ihre Beteiligung an transnationalen Verhandlungsprozessen dient vor allem der Stärkung ihrer Position in der jeweiligen nationalen Politik-Arena, indem beispielsweise der Mittel-Rückfluss aus Gemeinschaftsprojekten zum Hebel für Aufund Ausbau organisationaler Domänen wird. ${ }^{3}$ Die Gegenbewegungen sind zugleich unübersehbar: In der Raumfahrt beispielsweise gab es - bereits seit den 1960er Jahren und nunmehr verstärkt seit der Neuausrichtung der Raumfahrtpolitik der EU im Jahr 2000 - immer wieder Tendenzen, diese nationalstaatliche Ausrichtung durch eine weitere Ebene zu überlagern und die Zuständigkeiten auf europäischer Ebene zu zentralisieren. Aber dies ist ein langwieriger Prozess, der von widersprüchlichen Interessen, Rivalitäten und Konflikten geprägt ist und dessen Ausgang offen ist. Eindeutige Trends sind zum gegenwärtigen Zeitpunkt keineswegs auszumachen.

Der zweite Punkt, das beharrliche Festhalten staatlicher Akteure am Modell Großtechnik trotz der damit verbundenen - politischen wie technischen - Risiken lässt sich mit einem Bündel von Faktoren erklären: Bei Großtechnikprojekten ist eine überschaubare Zahl von Akteuren im Spiel; die Politik hat zudem einen exklusiven Zugriff auf die finanziellen, apparativen und personellen Ressourcen; sie kann das Vorhaben relativ leicht gegen Störungen von außen abschirmen; und schließlich spielen Unwägbarkeiten aufgrund von Markt-Turbulenzen nur eine geringe Rolle. Dies gilt insbesondere in innovativen Feldern, die von den Markt-Akteuren noch nicht besetzt sind und in denen sich noch keine industriellen Strukturen herausgebildet haben.

Politisch ist Großtechnik insofern eine nahe liegende Alternative, als hier zumindest die Illusion einer Kontrolle und Steuerung der Prozesse gewahrt werden kann, die sich zudem überwiegend innerhalb der eigenen PolitikDomäne abspielen bzw. an industrielle Akteure delegiert werden, die sich in einer engen Inte- 
ressenallianz mit den politischen Akteuren befinden (und daher keinen Anreiz haben, ihre Partner auf Seiten der Politik auf eventuelle Probleme und Fehlentwicklungen aufmerksam zu machen). Die Förderung großtechnischer Projekte trägt somit - trotz aller Brüche und Widersprüche - zum Bild eines aktivistischen, steuerungsmächtigen Staates bei, der in der Lage ist, systematisch Wirkungen zu erzeugen, die einzelnen (individuellen wie korporativen) Akteuren zugerechnet und daher in politische Gewinne umgemünzt werden können. (In marktnahen Bereichen ist ein Erzeugen von Wirkungen bzw. die Zurechnung von Effekten auf politisches Handeln weitaus schwieriger.) Zugleich gewährt der große Zeithorizont eine ausreichende Distanz, um sich der Verantwortung für mögliche Fehlschläge zu entziehen. Im Zweifelsfall sind für die „Flops“ immer die Vorgänger-Regierungen verantwortlich, oder man definiert die gescheiterten Vorhaben nachträglich zu Technologieprojekten um, die sich durch die damit verbundenen Lerneffekte und Kompetenzgewinne rechtfertigen lassen.

Auch die immer wieder auftretenden Kollateralschäden großtechnischer Projekte sind kein ernsthaftes Hindernis für ein staatliches Engagement; denn in der Bewältigung der teils durch eigenes Handeln verursachten „Folgeprobleme der Modernisierung“ (Beck) kann der Staat wiederum Profil zeigen, d. h. seine Handlungsfähigkeit unter Beweis stellen und zugleich die exklusive Kompetenz für diesen Politikbereich beanspruchen - was im Interesse des Domänenausbaus und der Legitimation staatlichen Handelns durchaus funktional ist. Trotz der damit verbundenen Risiken ist die staatliche Förderung von Großtechnik also eine attraktive Option, die die Handlungsfähigkeit staatlicher Akteure stärkt und ihre Macht und ihren Einfluss steigert. Die kurzfristigen institutionellen Effekte wiegen schwerer als die möglichen langfristigen Risiken und gesellschaftlichen Folgewirkungen, die ja ohnehin nicht die Politiker treffen, welche die Entscheidungen getätigt haben.

\section{Schlussbetrachtung: Koexistenz der Politikstile}

Historisch gesehen, war die symbiotische Beziehung von Politik und Technik einer der maßgeblichen Faktoren bei der Herausbildung des modernen Interventionsstaates; und strukturell betrachtet, bilden staatliche GroßtechnikProjekte eine wichtige Ressource für die Entfaltung und Stabilisierung politischer Macht. Aus diesen beiden Gründen spricht also wenig für die pauschale These eines Bedeutungsverlustes des Nationalstaates in der (Technologie-) Politik; auch die Vermutung, dass die staatlich inszenierte Entwicklung marktferner „Dinosaurier“ ein Auslaufmodell darstellt, das gegenwärtig von anderen Formen der Technologieförderung generell abgelöst werden wird, erweist sich als nicht stichhaltig.

Die Sachlage ist vielmehr komplizierter, und die Tendenzen sind vielschichtiger und $\mathrm{z}$. T. widersprüchlich. Erstens findet man in einigen Bereichen wie der Biotechnologie oder der Informationstechnik eine weit gehende Auflösung traditioneller Politikmodelle zugunsten neuer Governance-Stile wie etwa der Techniksteuerung durch Regulierung und/oder Partizipation (Werle 2001). In der Chemie- wie der Elektronikindustrie gibt es längst keine nationalen Champions mehr, auf die sich eine nationalstaatliche Politik ausrichten könnte; in der Industrie ist die Transnationalisierung wesentlich weiter fortgeschritten als in der Politik. Zweitens aber kehrt das klassische Politikmodell des Auf- und Ausbaus von Machtpositionen über Großtechnikprojekte (nebst der damit einhergehenden Zentralisierungstendenzen, vgl. Mayntz 2001) auf der nächst-höheren Ebene wieder, beispielsweise im Galileo-Projekt der EU. Drittens schließlich zeigt dieses Politikmodell auch im nationalstaatlichen Rahmen eine erstaunliche Persistenz, was sich am Beispiel der Lkw-Maut nachweisen lässt, wo in klassischer industriepolitischer Manier nationale Champions auf staatlich protektionierten Märkten gezüchtet werden.

Das Neben-, Mit- und teils Gegeneinander der verschiedenen Politikstile führt also zu einer neuen Unübersichtlichkeit in der Technologiepolitik. Institutionelle Innovationen finden zudem nicht in allen Bereichen zugleich statt; manche Innovation ist rein rhetorischer Natur. Die Technologiepolitik der entwickelten Industrienationen gleicht vielmehr einem „Gemischtwarenladen“, der durch die Koexistenz unterschiedlicher Stile und Modelle geprägt ist. Und hierbei spielt die staatliche Förderung von Großtechnik nach wie vor eine bedeutende Rolle, für die es - betrachtet man die politische 
Logik der hier gespielten Spiele - bislang kein funktionales Äquivalent gibt.

\section{Anmerkungen}

1) Eine ausführliche Rekonstruktion der drei Fallbeispiele findet sich in Weyer 2004 sowie im Preprint, das unter http://www.techniksoziologiedortmund.de/veroeffentlichung/files/ap3.pdf $\quad$ zu finden ist.

2) Dieser Ansatz steht in deutlichem Gegensatz zum Autopoiesis-Konzept von Niklas Luhmann und postuliert, dass die Logik der innersystemischen Spiele durch den Code nur unzureichend beschrieben wird; Handlungs- und Systemlogik fallen nicht in eins, sondern müssen mit unterschiedlichen Konzepten beschrieben werden.

3) Und umgekehrt wird eine starke nationale Position als Voraussetzung für ein selbstbewusstes Auftreten in internationalen Kontexten gesehen.

\section{Literatur}

$B M B F$ - Bundesministerium für Bildung und Forschung (Hrsg.), 2004: Bundesbericht Forschung 2004. Bonn

BMVBW - Bundesministerium für Verkehr, Bauund Wohnungswesen, 2005: Lkw-Maut ist gestartet. Pressemitteilung des BMVBW vom 01.01.2005; http://www.bmvbw.de/bmvbw-.302.22480/.htm (04.01.2005)

Bruzelius, N. et al., 2002: Big decisions, big risks. Improving accountability in mega projects. In: Transport Policy 9, S. 143-154

Bulmahn, E., 2002: Die strategische Bedeutung der Raumfahrt für Europa. In: DLR-Nachrichten 102 (April 2002), S. 2-5

Bulmahn, E., 2004: Raumfahrtforschung. Ein großer Nutzen für die Menschheit. In: Ignition, Sept. 2004, S. 2-7

Dolata, U., 2004: Unfassbare Technologie, internationale Innovationsverläufe und ausdifferenzierte Politikregime. Perspektiven nationaler Technologie- und Innovationspolitiken. Bremen (artec-paper Nr. 110)

Grande, E., 2001: Von der Technologie- zur Innovationspolitik - Europäische Forschungs- und Technologiepolitik im Zeitalter der Globalisierung. In: Simonis et al. 2001, S. 368-387

Hagen, R.; Scheffran, J., 2000: Weltraum - ein Instrument europäischer Macht. In: http://www.hsfk.de/ abm/print/forum/hagsch.htm (20.12.2002)

Keck, O., 1984: Der Schnelle Brüter. Eine Fallstudie zu Entscheidungsprozessen über Großtechnik. Frankfurt, New York: Campus
Keck, O., 1988: A theory of white elephants: Asymmetric information in government support for technology. In: Research Policy 17, S. 187-201

Mayntz, R., 2001: Triebkräfte der Technikentwicklung und die Rolle des Staates. In: Simonis 2001, S. 3-18

Reinke, N., 2004: Geschichte der deutschen Raumfahrtpolitik. Konzepte, Einflussfaktoren und Interdependenzen 1923-2002. München: R. Oldenburg

Simonis, G. et al. (Hrsg.), 2001: Politik und Technik. Analysen zum Verhältnis von technologischem, politischem und staatlichem Wandel am Anfang des 21. Jahrhunderts. Wiesbaden: Westdeutscher Verlag (PVS Sonderheft 31)

Werle, R., 2001: Liberalisierung und politische Techniksteuerung. In: Simonis 2001, S. 407-423

Weyer, J., 1992: Der Raumtransporter SÄNGER als Instrument deutscher Großmachtpolitik? Gutachten, erstellt im Auftrag des Büros für Technikfolgen-Abschätzung beim Deutschen Bundestag (Materialien zum TAB-Arbeitsbericht Nr. 14). Bonn (Oktober)

Weyer, J., 1993a: Akteurstrategien und strukturelle Eigendynamiken. Raumfahrt in Westdeutschland 1945-1965. Göttingen: Otto Schwartz

Weyer, J., 1993b: System und Akteur. Zum Nutzen zweier soziologischer Paradigmen bei der Erklärung erfolgreichen Scheiterns. In: Kölner Zeitschrift für Soziologie und Sozialpsychologie 45, S. 1-22

Weyer, J. et al., 1997: Technik, die Gesellschaft schafft. Soziale Netzwerke als Ort der Technikgenese. Berlin: edition sigma

Weyer, J., 1999: Wernher von Braun. Reinbek b. Hamburg: Rowohlt

Weyer, J., 2004: Innovationen fördern - aber wie? Zur Rolle des Staates in der Innovationspolitik. In: Rasch, M.; Bleidick, D. (Hrsg.): Technikgeschichte im Ruhrgebiet - Technikgeschichte für das Ruhrgebiet. Essen: Klartext Verlag, S. 278-294

\section{Kontakt}

Prof. Dr. Johannes Weyer

Universität Dortmund

Wirtschafts- und Sozialwissenschaftliche Fakultät 44221 Dortmund

Tel.: +49 (0) 231 / 755 - 3281

Fax: +49 (0) 231 / 755 - 3293

E-Mail: johannes.weyer@uni-dortmund.de Internet: http://www.wiso.uni-dortmund.de/TS 